九州大学学術情報リポジトリ

Kyushu University Institutional Repository

\title{
Portable and noise-tolerant magnetic field generation system
}

Edamoto, Masafumi

Interdisciplinary Graduate School of Engineering Sciences, Kyushu University

Morita, Taichi

Faculty of Engineering Sciences, Kyushu University

Saito, Naoya

Interdisciplinary Graduate School of Engineering Sciences, Kyushu University

Itadani, Yutaro

Interdisciplinary Graduate School of Engineering Sciences, Kyushu University

他

http://hdl. hand le. net/2324/1956706

出版情報 : Review of Scientific Instruments. 89, pp.094706-1-094706-5，2018-09-24. AIP Publishing

バージョン :

権利関係 : 


\title{
Portable and noise-tolerant magnetic field generation system
}

\author{
Masafumi Edamoto, ${ }^{1, \text { a) }}$ Taichi Morita, ${ }^{2}$ Naoya Saito, ${ }^{1}$ Yutaro Itadani, ${ }^{1}$ Satoshi Miura, ${ }^{1}$ \\ Shinsuke Fujioka, ${ }^{3}$ Hideki Nakashima, ${ }^{2}$ and Naoji Yamamoto ${ }^{2}$ \\ 1) Interdisciplinary Graduate School of Engineering Sciences, Kyushu University, \\ Kasuga, 816-8580, Japan \\ ${ }^{2)}$ Faculty of Engineering Sciences, Kyushu University, Kasuga, 816-8580, \\ Japan \\ 3) Institute of Laser Engineering, Osaka University, Suita, 565-0871, \\ Japan
}

(Dated: 3 September 2018)

We have successfully developed a portable pulsed magnetic field generation system incorporating a number of techniques to avoid the effects of noise, including shielding, a self-power capability, and a high-capability semiconductor switch. The system fits into a cubical box less than $0.5 \mathrm{~m}$ in linear dimensions and can easily be installed in experimental facilities, including noisy environments such as high-power laser facilities. The system can generate a magnetic field of several tesla sustainable for several tens of microseconds over a spatial scale of several centimeters. In a high-power laser experiment with Gekko-XII, the system operated stably despite being subjected to a high level of electrical noise from laser shots of $600 \mathrm{~J}$.

PACS numbers: 07.55.Db, 07.50.Ek, 84.30.Jc, 52.50.Jm

Keywords: Magnetic field generation, Pulsed power device, Plasma control, Laser ablation plasma

a)edamoto@aees.kyushu-u.ac.jp 
Portable and noise-tolerant magnetic field generation system

\section{INTRODUCTION}

Interactions between plasmas and magnetic fields are of great importance in both scientific and engineering researches. In the scientific field, the availability of high-power lasers and techniques for generating strong magnetic fields has led to a number of advances in laboratory astrophysics, including experimental studies of collisionless shock waves ${ }^{1}$ and magnetic reconnection. ${ }^{2}$ Such investigations require a range of magnetic fields from 1 to $1000 \mathrm{~T}$ on time scales of nanoseconds to microseconds and spatial scales of millimeters to centimeters. In engineering applications, some types of fusion reactors and plasma propulsion thrusters use magnetic fields to control plasmas. Plasma confinement ${ }^{3}$ requires magnetic fields of tens of tesla sustained for tens of nanoseconds within a submillimeter-scale region. Magnetic nozzles for advanced rocket systems (laser fusion rockets) require magnetic fields of several tesla sustained for several milliseconds in a meter-scale region for flight models ${ }^{4}$ and fields of several tesla for tens of microseconds in a centimeter-scale region for scaled-down experimental models. ${ }^{5,6}$ In previous research, kilotesla magnetic fields were generated using a capacitor-coil target and a high-power laser beam. ${ }^{7}$ This method is able to produce a sharp pulse of quite high magnetic field in a small volume, but the pulse width is only a few nanoseconds. The capacitor-coil target is destroyed by irradiation at each laser shot. In a nondestructive approach, coils powered by capacitor banks have been widely used. Magnetic

fields of several tens of tesla have been achieved, ${ }^{8-14}$ but a large power source is required to generate a long-duration pulsed magnetic field. It is impossible to transport such power sources between experimental facilities owing to their huge mass and volume.

It is generally not feasible for each large laser facility to have its own dedicated equipment for the generation of large magnetic fields, because most are joint user facilities, and therefore a portable magnetic field generation system is highly desirable. Noise tolerance is also required, because each high-power laser shot generates a large amount of electrical noise. In previous experiments, ${ }^{15}$ the semiconductor switch of the capacitor bank was frequently damaged.

In this paper, we present a portable and noise-tolerant magnetic field generation system for high-power laser experiments. The target strength of the magnetic field is several tesla for tens of microseconds in a region with dimensions of several centimeters. The time scale of the laser-produced plasma is quasi-steady. The criterion for portability is that the equipment 
Portable and noise-tolerant magnetic field generation system

should be capable of being transported by a single person.

\section{DRIVING CIRCUIT}

Figure 1 shows the circuit overview of the capacitor bank for magnetic field generation. $S W 1, S W 2$, and $S W 3$ are manual switches, and $S W 4$ is a semiconductor switch that is controllable by an external trigger. The circuit has five modes, depending on the connections and the charged voltage of the capacitor, as listed in Table I: "charging," "waiting," "driving," "dumping," and "safe." In the charging mode, the DC power source $V(0-700 \mathrm{~V}, 10 \mathrm{~mA})$ and the capacitor $C$ (FFLI6B3007K-- film capacitor, $3000 \mu \mathrm{F}$ ) are connected through the resistor $R_{\text {charge }}(20 \mathrm{k} \Omega, 5 \mathrm{~W})$ and the switches $S W 1$ and $S W 2$ to charge the capacitor. When the capacitor voltage reaches a target value, $S W 1$ and $S W 2$ are opened and the circuit shifts to the waiting mode. The breaking of the connection between the power source and the capacitor bank on both the positive and negative potentials by using $S W 1$ and $S W 2$, respectively, protects the power source from rapid changes in the load impedance and from surge noise from the coil and the ground. In the waiting mode, the circuit waits for an external trigger to turn on the switch $S W 4$ to drive the coil. When the circuit receives a trigger, it shifts to the driving mode. Then, the discharge current from the capacitor flows through the coil $L(8.8 \mu \mathrm{H}$ including the connecting wire) to generate a magnetic field. After the discharge, $S W 4$ is turned off automatically. Then, the circuit shifts to the dumping mode by closing SW3 and the remaining electrical energy of the capacitor is consumed by the resistor $R_{\text {dump }}$ $(1 \mathrm{k} \Omega, 1 \mathrm{~kW})$. When the voltage of the capacitor becomes almost zero, the circuit shifts to the safe mode.

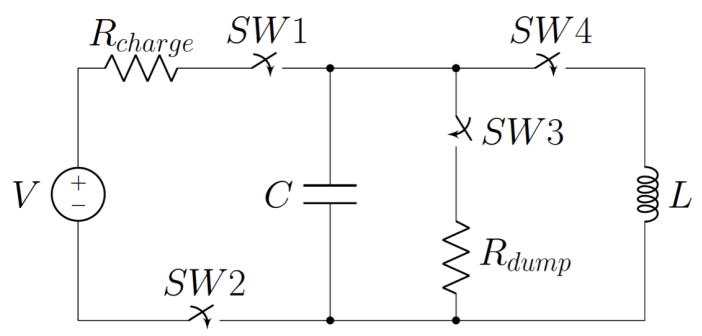

FIG. 1. Circuit overview of the magnetic field generation system.

In our previous experiments, ${ }^{15}$ we used an insulated gate bipolar transistor (IGBT) module as the switch $S W 4$, but this had low reliability, especially on high-power (>300 J) laser 
Portable and noise-tolerant magnetic field generation system

TABLE I. Modes of the circuit.

\begin{tabular}{|c|c|c|c|c|}
\hline Mode & $S W 1$ & $S W 2$ & $S W 3$ & $S W 4$ \\
\hline \hline Charging & ON & ON & OFF & OFF \\
\hline Waiting & OFF & OFF & OFF & OFF \\
\hline Driving & OFF & OFF & OFF & ON \\
\hline Dumping / Safe & OFF & OFF & ON & OFF \\
\hline
\end{tabular}

shots, even though a snubber circuit was installed to protect it from surge noise. The module worked stably in experiments with $60 \mathrm{~J}$ laser shots $(10 \mathrm{~J} \times$ six beams). However, it broke every two or three times in experiments with $300 \mathrm{~J}$ shots $(50 \mathrm{~J} \times$ six beams $)$, and every time with $600 \mathrm{~J}$ shots $(100 \mathrm{~J} \times$ six beams). These failures were probably caused by the counter-electromotive force of the coil. The plasma generated by laser irradiation of a solid sphere expands in an external magnetic field. Then, a diamagnetic current is generated in the plasma, deforming the external magnetic field. The rapid change in the magnetic field is picked up by the coil, and the coil generates an inverse voltage. It is this inverse voltage that can damage the module.

Here, we used a thyristor because it has many advantages for a pulsed power system. First, the voltage capability of a thyristor is high for both forward and backward voltage. Oscillations in the $L C$ circuit and the counter-electromotive force apply an inverse high voltage to the switching device. Second, a thyristor will hold its turned-on state owing to its forward current. When the switching device turns off suddenly, the coil generates a high voltage as a counter-electromotive force. Because of its self-holding capability, a thyristor will decrease the possibility of such sudden disconnects. We used a capsule thyristor (DCR860D18). As shown in Fig. 2, it was sandwiched between copper plates and carbon sheets. Its maximum ratings were $1.8 \mathrm{kV}$ and $860 \mathrm{~A}$ for a continuous current, and $11.5 \mathrm{kA}$ for a surge current (a surge current is defined here as a half sine wave of frequency $1 \mathrm{kHz}$ ).

Figure 3 shows the driver circuit for the thyristor. The circuit receives an external trigger from a delay generator and drives the thyristor. In this driver circuit, several methods are adopted to prevent malfunctions caused by large laser shot noise and the high-power switching. First, together with other systems, the circuit is enclosed within an aluminum box to shield it from radiation noise. The box is electrically floating condition to avoid 


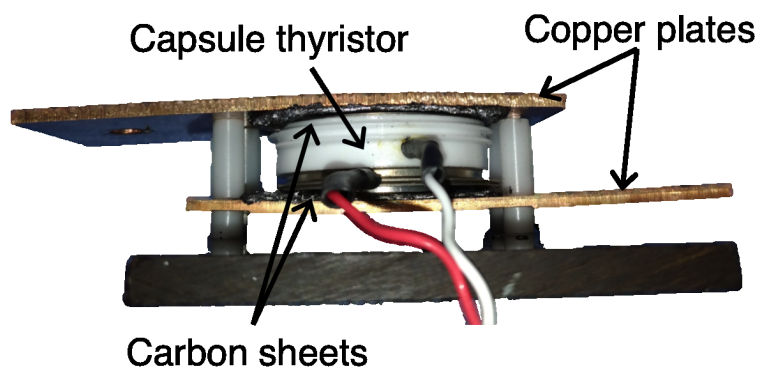

FIG. 2. The thyristor module, with the capsule thyristor sandwiched between copper plates and carbon sheets.

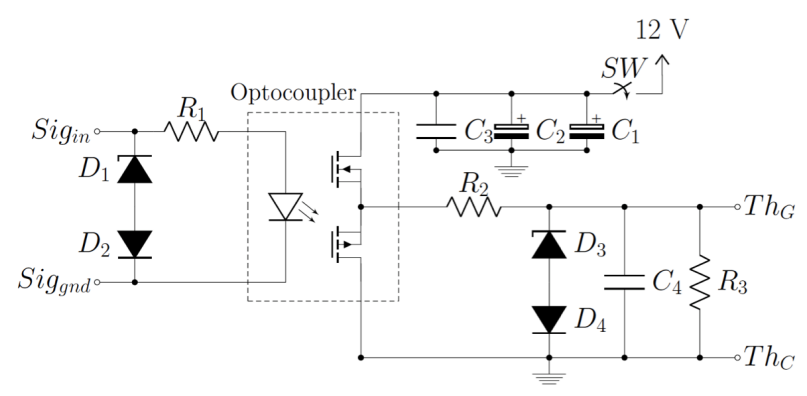

FIG. 3. The self-powered thyristor driver circuit.

any electrical noise conducted from the ground. Second, clipping Zener diodes $D_{1}$ and $D_{3}$ are used to protect the circuit from radiation noise picked up by cables extending outside the aluminum box. Third, the external trigger signal is transmitted through a totem pole optocoupler (TLP250H). This optocoupler protects the trigger source from any unexpected direct connection of the high-power line and the signal line. Such a connection might occur if the thyristor is severely damaged. In addition, the totem pole circuit prevents the occurrence of a high-impedance condition, because it keeps the voltage of the signal line at zero until a trigger arrives. Fourth, the circuit is separated from the external power source before each laser shot and is operated by a super-capacitor $C_{1}$ and a chemical capacitor $C_{2}$ to avoid the noise that would arise from external power cables. The super-capacitor is used to maintain the voltage of the chemical capacitor sufficiently high during the waiting mode, and the chemical capacitor is used to drive the thyristor. The super-capacitor consists of three $1 \mathrm{~F}$ electric double-layer capacitors connected in series. During operation, the capacitors are charged up to $13 \mathrm{~V}$. The voltage decreases with time owing to self-discharge and falls below the lower working limit, $10 \mathrm{~V}$, after 10 minutes. This is acceptable, since the time required 
for typical laser experiments on the Gekko-XII (GXII) laser facilities at Osaka University is only a few minutes. The circuit was implemented on a printed circuit board.

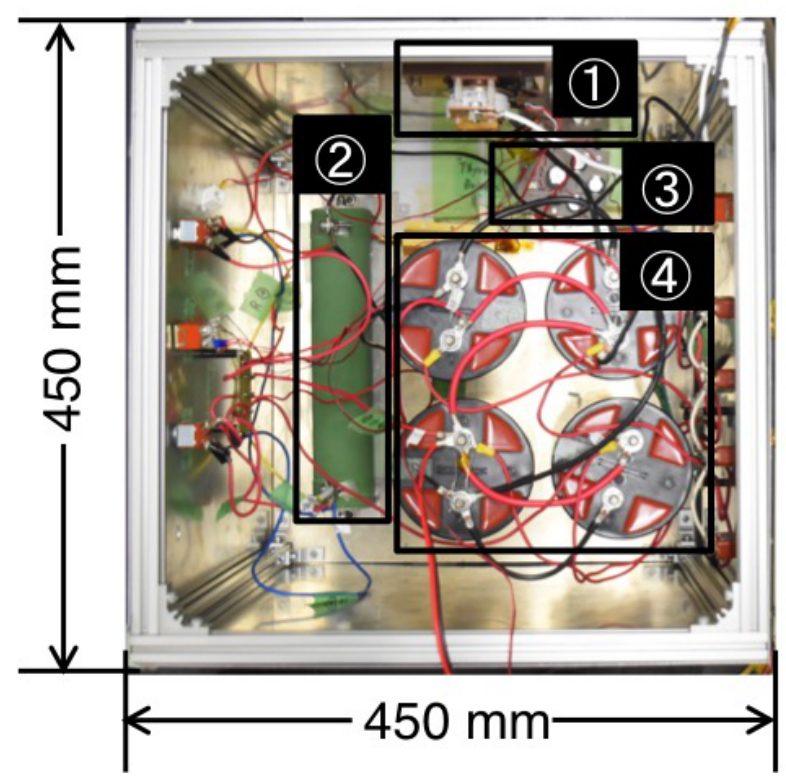

FIG. 4. Top view of the capacitor bank: (1) thyristor; (2) $R_{\text {dump }}$; (3) trigger circuit; (4) capacitors.

Figure 4 shows the assembled capacitor bank. The outer case consists of extruded aluminum profiles and plates. The thyristor is positioned on one of the side walls, with a Bakelite board insulating it from the aluminum case. The driver circuit is positioned on the bottom, and the external trigger line is connected through a BNC connector. The enameled resistor $R_{\text {dump }}$ is also positioned on the bottom and is connected to the capacitors through the switch $S W 3$. All the switches are positioned on one of the side walls. The four cylindrical film capacitors are fixed to the bottom by screws. The dimensions of the capacitor bank as a whole are $450 \mathrm{~mm}$ (width) $\times 450 \mathrm{~mm}$ (length) $\times 500 \mathrm{~mm}$ (height)

We conducted a standalone experiment to test the circuit shown in Fig. 5. In this experiment, the coil consisted of a polyimide-insulated flat copper wire of thickness $0.4 \mathrm{~mm}$ and width $5 \mathrm{~mm}$ and an aluminum coil core of inner diameter $41 \mathrm{~mm}$ with 10 turns in the radial direction. The inductance of the coil was $4.5 \mu \mathrm{H}$. The inductance was measured by an $L C R$ meter at a measurement frequency of $1 \mathrm{kHz}$. The coil and the capacitor bank were connected by a several meters of twisted conducting wire of cross-sectional area $3.5 \mathrm{~mm}^{2}$ and a copper feed-through. The wire and the feed-through were connected by a crimped terminal and a beryllium copper coupler. All connection points except the trigger circuit were connected 
with solderless terminals. The total inductance of the capacitor bank including the wire and the feed-through was $8.8 \mu \mathrm{H}$.

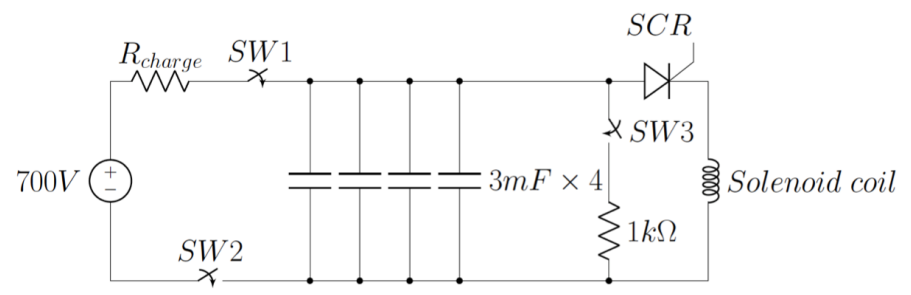

FIG. 5. The circuit for the standalone experiment: the four capacitors are connected in parallel.

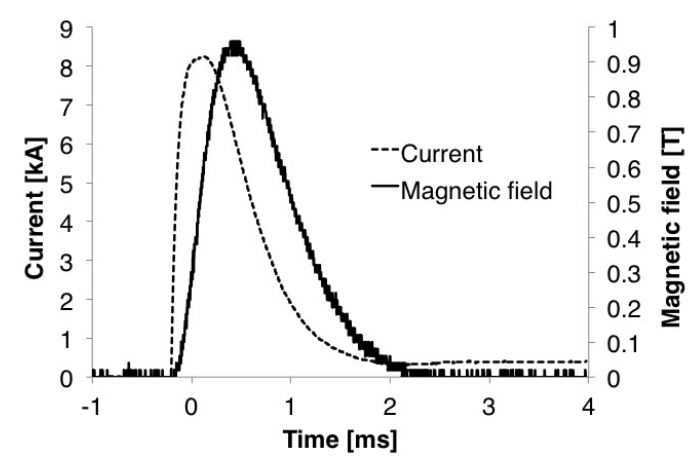

FIG. 6. Magnetic field strength and coil driving current in the standalone experiment. The magnetic field was delayed due to an internal delay and a rise time of the Gauss meter.

Figure 6 shows the coil current and the magnetic field, as measured using a Rogowski coil and a Gauss meter (Model: 5180 produced by F. W. BELL), respectively. The magnetic field was measured at a distance of $11 \mathrm{~mm}$ from the coil surface along the coil axis. The measured magnetic field shows slower time-variation compared with the current because of an internal delay of $30 \mu \mathrm{s}$ and a rise time of $250 \mu \mathrm{s}$ in the Gauss meter. The full pulse width of the magnetic field was approximately $2 \mathrm{~ms}$. The magnetic field was assumed to be in a quasi-steady state around the peak of the pulse up to a few tens of microseconds, which is sufficiently long compared with the typical time scale of plasma expansion, which is a few microseconds.

\section{HIGH-POWER LASER EXPERIMENT}

Using the system described in the previous section, we conducted an experiment with the GXII high-power laser to investigate the interaction between a laser-produced plasma 
Portable and noise-tolerant magnetic field generation system

and an external magnetic field. Figure 7 shows the top view of the experimental setup. The circuit setup and the parameters were the same as for the standalone experiment. A plasma was generated by irradiating a spherical polystyrene $(\mathrm{CH})$ shell of outer diameter $500 \mu \mathrm{m}$ and thickness $7 \mu \mathrm{m}$ positioned at the center of a vacuum chamber. The target was irradiated by six beams of the high-power laser. The wavelength and pulse width were $1053 \mathrm{~nm}$ and $1.3 \mathrm{~ns}$ (FWHM), respectively. We conducted several laser shots with different output laser energies of 25, 50, and $100 \mathrm{~J} /$ beam. The distance between the target and the surface of the coil was $11 \mathrm{~mm}$, as shown in Fig. 7.

We measured thermal bremsstrahlung from the laser-produced plasma along the measurement line shown in Fig. 7 to estimate the density distribution of the plasma. The coil driving current was also measured, using a Rogowski coil.

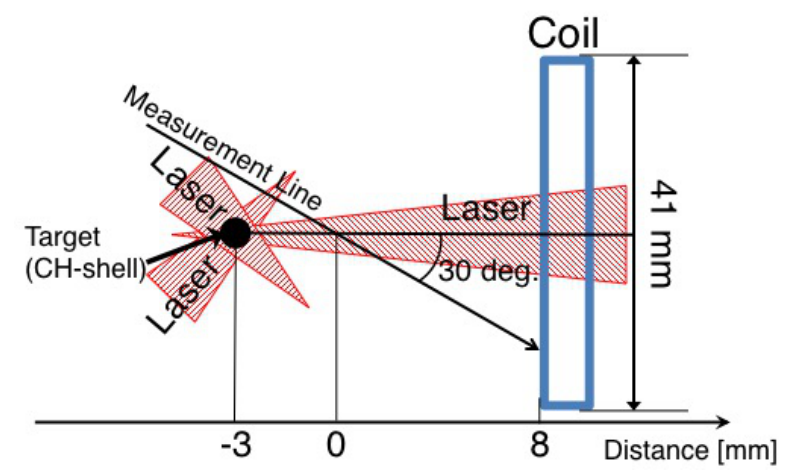

FIG. 7. Top view of the experimental setup for the laser experiment

We have already achieved magnetic field generation and plasma control using the setup shown in Fig. 7 in previous research, ${ }^{16}$ in which we used an IGBT instead of a thyristor as the switch $S W 4$. Figure 8 shows the spatial distribution of the electron density measured in that experiment. The six laser beams with energy $100 \mathrm{~J} /$ beam irradiated the target in the external magnetic field of $1 \mathrm{~T}$ at the original target position. The horizontal and vertical axes are the axial and radial directions of the coil, respectively. $r=0$ is the coil axis, and the original target position was $(x, r)=(-3,0)$. These data were obtained from interferometric observations and calculated using the Abel inversion method. The interferometric observations measured the difference of refractive indices in the plasma. The plasma pressure and magnetic field pressure are balanced in the high-density shell-like regions. At this time, the IGBT was broken.

Figure 9 shows the current measured with a Rogowski coil with a charge voltage of $700 \mathrm{~V}$ in 


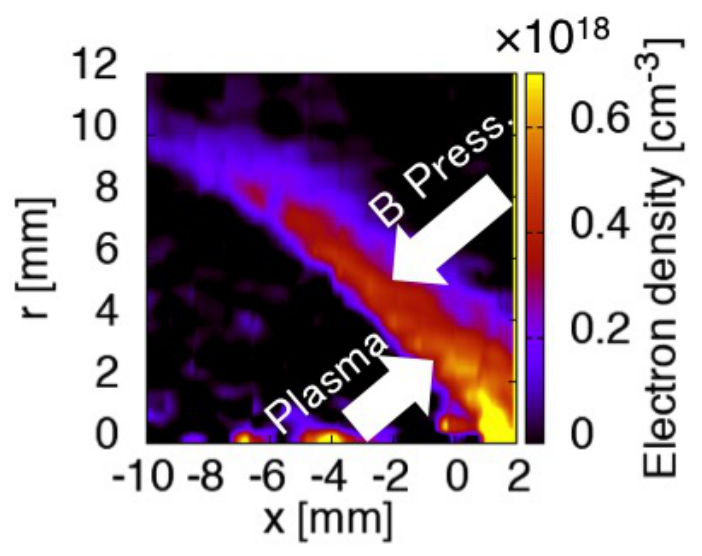

FIG. 8. Suppression of plasma expansion by an external magnetic field in the previous experiment.

the present experiment. At the time of peak current, six laser beams with energy $100 \mathrm{~J} / \mathrm{beam}$ irradiated the target to generate a quasi-spherically expanding plasma. The current shows a sharp drop at the instant of the laser shot. This might be due to a counter-electromotive force from the coil. Here, this does indeed seem to be the case, since another channel of the oscilloscope that was open measured no specific signals. The counter-electromotive force would have been generated by picking up the temporal variation of the magnetic field. This temporal variation was due to plasma expansion. In this series of experiments, we performed ten stable laser shots with this system, with different energies of 25, 50, and $100 \mathrm{~J} /$ beam. The magnetic field generation system worked well for every shot, and a check of operational success after the series of experiments revealed no failures. We checked the magnetic field generation system step by step along the mode transitions shown in Table I. The coil swelled as a result of hoop stress induced by the magnetic field generation, and therefore the electrical parameters such as inductance, resistance, and capacitance should be changed slightly. However, the driving current and the generated magnetic field strength were almost the same as those found from the standalone experiment.

We checked the magnetic field generation in the high-power laser experiment from observations of the plasma behavior. Figure 10 shows the averaged intensity of the light emission from the laser-produced plasma in the wavelength range $529 \mathrm{~nm} \leq \lambda \leq 531 \mathrm{~nm}$, where there are no specific emission or absorption lines. The charge voltage was $500 \mathrm{~V}$ in the every case on this figure. The data obtained at all the different laser energies indicate that thermal bremsstrahlung is dominant in this wavelength range. In the case of a driving laser energy 
Portable and noise-tolerant magnetic field generation system

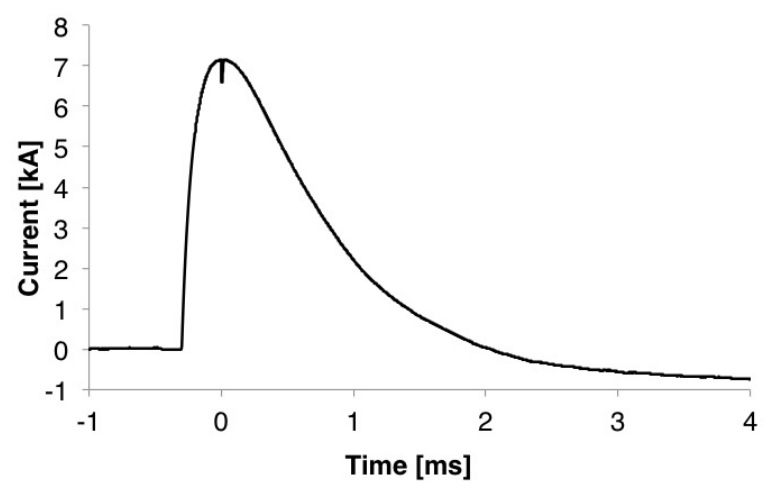

FIG. 9. Coil driving current from the experiment with a laser-produced plasma.

of $25 \mathrm{~J} /$ beam, the plasma stagnated at a distance of 5-6 $\mathrm{mm}$ from the target owing to interaction with the external magnetic field. On the other hand, for higher laser energies, the plasma did not stagnate significantly. This shows that the magnetic field energy is not sufficient to stagnate plasmas produced by laser energies greater than $50 \mathrm{~J} / \mathrm{beam}$.

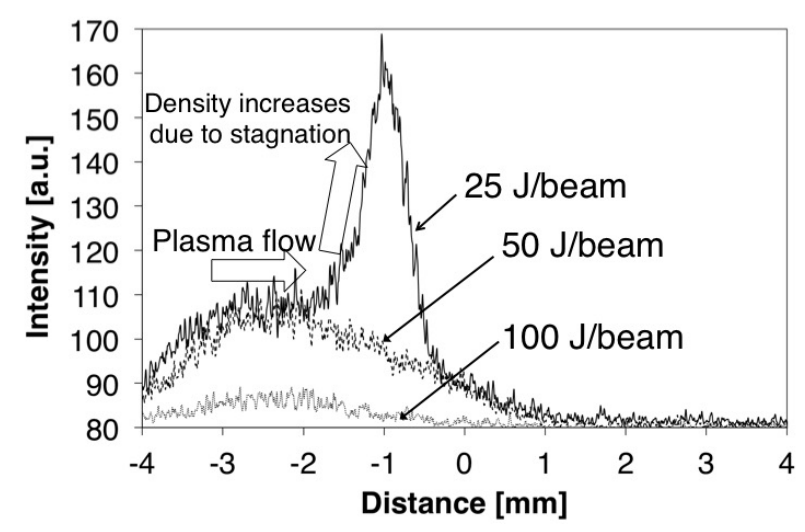

FIG. 10. Averaged intensity of thermal bremsstrahlung emission in the wavelength range $529 \mathrm{~nm}$ $\leq \lambda \leq 531 \mathrm{~nm}$ versus distance.

\section{CONCLUSION}

We have developed a portable and noise-tolerant magnetic field generation system that is capable of producing a magnetic field of $1 \mathrm{~T}$ at a point $11 \mathrm{~mm}$ from the coil surface for several tens of microseconds. In our previous system, the failure rate was almost $100 \%$ with high-power laser shots of $600 \mathrm{~J}$. In contrast, with our new system, there were no failures at this laser energy. 
Portable and noise-tolerant magnetic field generation system

Several improvements are planned for the future. First, we intend to upgrade the capacitor bank to a multiline arrangement to drive a multicoil system. A means for adjusting the switching time of the semiconductor switch will therefore be needed to achieve synchronization with high temporal accuracy. Second, we aim to develop a pulse-forming circuit to allow the generation of long-duration pulses. This will require a compact inductance system to enable portability.

\section{ACKNOWLEDGMENTS}

The authors acknowledge the dedicated technical support by the staff at the GXII laser facilities. This work was supported by the Japan Society for the Promotion of Science (JSPS) KAKENHI (Grant Nos. 15K18283 and 17K14876) and by the Joint Research Project of the Institute of Laser Engineering, Osaka University.

\section{REFERENCES}

${ }^{1}$ D. B. Schaeffer, W. Fox, D. Haberberger, G. Fiksel, A. Bhattacharjee, D. H. Barnak, S. X. Hu, and K. Germaschewski. Phys. Rev. Lett., 119:025001, 2017.

${ }^{2}$ X. X. Pei, J. Y. Zhong, Y. Sakawa, Z. Zhang, K. Zhang, H. G. Wei, Y. T. Li, Y. F. Li, B. J. Zhu, T. Sano, Y. Hara, S. Kondo, S. Fujioka, G. Y. Liang, F. L. Wang, and G. Zhao. Physics of Plasmas, 23(3):032125, 2016.

${ }^{3}$ H. Tang, G. Hu, Y. Liang, T. Tao, Y. Wang, P. Hu, B. Zhao, and J. Zheng. Plasma Physics and Controlled Fusion, 60(5):055005, 2018.

${ }^{4}$ Y. Nagamine and H. Nakashima. Fusion Technology, 35(1):62, 1999.

${ }^{5}$ A. Maeno, T. Hinaga, N. Yamamoto, A. Sunahara, S. Fujioka, and H. Nakasima. Journal of Propulsion and Power, 30(1):54, 2013.

${ }^{6}$ T. Morita, M. Edamoto, S. Miura, A. Sunahara, N. Saito, Y. Itadani, T. Kojima, Y. Mori, T. Johzaki, Y. Kajimura, S. Fujioka, A. Yogo, H. Nishimura, H. Nakashima, and N. Yamamoto. Scientific Reports, 7(1):8910, 2017.

${ }^{7}$ S. Fujioka, Z. Zhang, K. Ishihara, K. Shigemori, Y. Hironaka, T. Johzaki, A. Sunahara, N. Yamamoto, H. Nakashima, T. Watanabe, H. Shiraga, H. Nishimura, and H. Azechi. Scientific Reports, 3:1170, 2013. 
Portable and noise-tolerant magnetic field generation system

${ }^{8}$ B. B. Pollock, D. H. Froula, P. F. Davis, J. S. Ross, S. Fulkerson, J. Bower, J. Satariano, D. Price, K. Krushelnick, and S. H. Glenzer. Review of Scientific Instruments, $77(11): 114703,2006$.

${ }^{9}$ O. V. Gotchev, J. P. Knauer, P. Y. Chang, N. W. Jang, M. J. Shoup, D. D. Meyerhofer, and R. Betti. Review of Scientific Instruments, 80(4):043504, 2009.

${ }^{10} \mathrm{~S}$. Zherlitsyn, B. Wustmann, T. Herrmannsdorfer, and J. Wosnitza. IEEE Transactions on Applied Superconductivity, 22(3):4300603-4300603, 2012.

${ }^{11}$ B. Albertazzi, J. Béard, A. Ciardi, T. Vinci, J. Albrecht, J. Billette, T. Burris-Mog, S. N. Chen, D. Da Silva, S. Dittrich, T. Herrmannsdörfer, B. Hirardin, F. Kroll, M. Nakatsutsumi, S. Nitsche, C. Riconda, L. Romagnagni, H.-P. Schlenvoigt, S. Simond, E. Veuillot, T. E. Cowan, O. Portugall, H. Pépin, and J. Fuchs. Review of Scientific Instruments, 84(4):043505, 2013.

${ }^{12}$ G. Fiksel, A. Agliata, D. Barnak, G. Brent, P.-Y. Chang, L. Folnsbee, G. Gates, D. Hasset, D. Lonobile, J. Magoon, D. Mastrosimone, M. J. Shoup, and R. Betti. Review of Scientific Instruments, 86(1):016105, 2015.

${ }^{13}$ G. Hu, Y. Liang, F. Song, P. Yuan, Y. Wang, B. Zhao, and J. Zheng. Plasma Science and Technology, 17(2):134, 2015.

${ }^{14}$ F. Duc, X. Tonon, J. Billette, B. Rollet, W. Knafo, F. Bourdarot, J. Béard, F. Mantegazza, B. Longuet, J. Lorenzo, Eddy Lelièvre-Berna, and L. Regnault P. Frings. Review of Scientific Instruments, 89(5):053905, 2018.

${ }^{15}$ R. Kawashima, T. Morita, N. Yamamoto, N. Saito, S. Fujioka, H. Nishimura, H. Matsukuma, A. Sunahara, Y. Mori, T. Johzaki, and H. Nakashima. Plasma and Fusion Research, 11:3406012, 2016.

${ }^{16}$ T. Morita, N. Yamamoto, R. Kawashima, N. Saito, M. Edamoto, S. Fujioka, Y. Itadani, T. Johzaki, S. Miura, and Y. Mori. Journal of Physics: Conference Series, 717(1):012071, 2016. 